\title{
DDX5/p68 RNA helicase expression is essential for initiating adipogenesis
}

\author{
Nardev Ramanathan ${ }^{1,3^{*}}$ (D) Nicole Lim ${ }^{1,2}$ and Colin L. Stewart ${ }^{1,2}$
}

\begin{abstract}
Background: DDX5/p68 RNA helicase is a member of the DEAD (Asp-Glu-Ala-Asp) box proteins. Apart from RNA unwinding, DDX5 is an important transcriptional factor and co-activator in cell proliferation and differentiation.

Findings: Here, we have characterised the role of DDX5 in adipogenesis in 3T3-L1 cells using siRNA mediated suppression. Transient inhibition of Ddx5 mRNA expression at the start of adipogenesis impairs the differentiation programme even when DDX5 expression is restored later in adipogenesis. However transient suppression of Ddx5 at the later stages of adipogenesis do not impair adipogenesis or triglyceride accumulation suggesting $D d \times 5$ expression is dispensable in a mature adipocyte.
\end{abstract}

Conclusion: These results implicate DDX5 as a crucial factor involved in the complex transcriptional cascade of events that regulate adipogenesis and essential to the initiation of adipogenesis.

Keywords: DDX5/p68, Adipogenesis, RNA helicase

\section{Introduction}

DEAD (Asp-Glu-Ala-Asp) Box Protein 5 (DDX5), or p68 RNA helicase is a multifunctional nuclear factor, first identified by its immunological cross-reactivity to a monoclonal antibody to the large $\mathrm{T}$ antigen of simian virus 40 [1]. DDX5 is a prototypical member of the DEAD/H-box protein family. The DEAD box family is characterized by a region of nine conserved amino acid motifs including Asp-Glu-Ala-Asp (DEAD) which are required in cellular functions such as pre-mRNA processing and ribosome biogenesis [2]. Co-purification of DDX5 with spliceosomes suggested a role in RNA splicing and this was subsequently confirmed when DDX5 was shown to be an essential splicing protein acting at the U1 snRNA-5' splice site [3]. More recently, DDX5 has been reported to co-activate several transcription factors in proliferation and differentiation that are themselves highly regulated, including the estrogen receptor $\alpha(E R \alpha)$, tumour suppressor $\mathrm{p} 53$, androgen receptor (AR), myogenic regulator MyoD, and the osteoblast

\footnotetext{
* Correspondence: nardevr@gmail.com

'Developmental and Regenerative Biology Laboratory, Institute of Medical Biology, \#06-06, 8A Biomedical Grove, Singapore 138648, Republic of Singapore

${ }^{3}$ Present address: Davos Life Science Pte Ltd, 3 Biopolis Drive, \#04-19, Synapse, Singapore 138623, Republic of Singapore

Full list of author information is available at the end of the article
}

differentiation factor Runx2 [4]. DDX5 has been hinted at to be involved in adipogenesis in one report [5], although with rudimentary characterisation. Here we sought to clarify the requirement of DDX5 in adipogenesis using a transient knockdown strategy.

\section{Materials and methods}

Cell culture and adipogenesis

3T3-L1 murine preadipocytes and C3H10T1/2 murine mesenchymal stem cells were cultured in high glucose (4500 mg/L) Dulbecco's modified Eagle medium (DMEM) supplemented by $10 \%$ heat-inactivated Newborn Calf Serum (NCS) (Sigma-Aldrich N4637) as described [6]. Two days after reaching $100 \%$ confluence, cells were induced to differentiate into adipocytes in high glucose DMEM supplemented with 10 \% Fetal Calf Serum (FCS) as described [6]. Oil Red O staining of lipid accumulation was performed as described [6] and triglyceride levels were quantified using a triglyceride quantification kit (Abcam, ab65336) according to the manufacturer's protocol.

\section{RNA isolation and quantitative real-time PCR (qRT-PCR)}

RNA extraction and purification was done using the RNeasy Mini Kit (Qiagen, \#74106) together with DNase digestion using the RNase-free DNase digestion kit 
(Qiagen, \#79254) and was performed according to the manufacturer's instructions. Purified RNA was reverse transcribed as previously described [7]. Reactions were performed using either Fast Taqman ${ }^{\circ}$ master mix with Taqman $^{\circ}$ primers or probes (ABI Biosystems) or $\mathrm{SYBR}^{\circ}$ green master mix (ABI Biosystems) and primers (Table 1). Expression of all the genes were normalized to the housekeeping gene Cyclophilin A (CycloA).

\section{Western blot analysis}

Cell lysis and western blotting was performed as described [7]. Antibodies used for western blotting were against PPARY (E8)(Santa Cruz, sc-7273, 1:1000), DDX5 (PAb204, kind gift from Frances-Fuller Pace, University of Dundee, 1:500) and GAPDH (FL-335, sc-25778, 1:2000).

\section{siRNA mediated suppression of gene expression}

Cells were treated with $20 \mu \mathrm{m}$ of either a control nontargeting siRNA or SMARTpool: ON-TARGETplus (GE Lifesciences) siRNA targeted against Ddx5, Pparg or Cebpb (Table 2) using Lipofectamine RNAiMAX (Life Technologies) as described [8].

\section{Cell viability assay}

Cells were treated with WST-1 reagent using the WST-1 Cell Viability Assay Kit (Cayman Chemicals, \#10008883) according to the manufacturer's protocol. Briefly, preadipocytes were seeded at a density of $10^{4}$ cells $/ \mathrm{cm}^{2}$ in a 96-well plate in triplicates. Cells were normalized to untreated cells. Bleomycin (Sigma-Aldrich, B8416) was used as a negative control (cytotoxic agent) at $200 \mu \mathrm{g} / \mathrm{ml}$. Both siRNA treatment and bleomycin treatment were administered for $48 \mathrm{~h}$.

\section{Results}

DDX5 expression in differentiating adipocytes- We investigated the expression of DDX5 during adipogenesis in two widely used preadipocytic lines, 3T3-L1 and C3H10T1/2 mesenchymal stem cells. We differentiated these lines for 8 days in vitro and assessed the mRNA and protein expression of DDX5. We observed that the mRNA expression of $D d x 5$ in 3T3-LI cells peaked at day 2 of differentiation (Fig. 1a) whereas $D d x 5$ was most strongly expressed before adipogenic induction (D0) in C3H10T1/2 cells (Fig. 1b). However, at the protein level, DDX5 peaked at D2 of differentiation in both these

Table 1 SYBR green primers used for quantitative real-time PCR

\begin{tabular}{lll}
\hline Gene & Forward primer & Reverse primer \\
\hline CycloA & TTCCTCCTTCACAGAATTATTCCA & CCGCCAGTGCCATTATGG \\
Pparg & GATGCACTGCCTATGAGCACT & AGAGGTCCACAGAGCTGATTC \\
Cebpb & AAGCTGAGCGACGAGTACAAGA & GTCAGCTCCAGCACCTTGTG \\
Lmna & GGAGGAGCTTGACTTCCAGAAG & CCACAAGCCGCGTCTCAT \\
\hline
\end{tabular}

Table 2 The SMARTpool siRNA ON-TARGETplus mouse siRNA (Thermo Scientific) designed to specifically target mouse Ddx5, Pparg and Cebpb. The SMARTpool siRNA consists of four individual duplexes designed to obtain a high level of gene silencing. The non-targeting siRNA is a negative control siRNA with at least four mismatches to any human mouse or rat gene

\begin{tabular}{|c|c|c|}
\hline siRNA & siRNA details & Target sequence \\
\hline \multirow[t]{4}{*}{$D d \times 5$ (13207) } & $D d \times 5$-J-065333-09 & $\overline{\text { CAGUAAAGUUUUCGGGUUA }}$ \\
\hline & Ddx5 -J-065333-10 & AAAUAAGACCUGAUAGGCA \\
\hline & Ddx5 -J-065333-11 & GCUGAAUAUUGUCGAGCUU \\
\hline & Ddx5 -J-065333-12 & ACAUAAAGCAAGUGAGCGA \\
\hline \multirow[t]{4}{*}{ Pparg (19016) } & Pparg -J-040712-05 & CGAAGAACCAUCCGAUUGA \\
\hline & Pparg -J-040712-06 & ACCCAAUGGUUGCUGAUUA \\
\hline & Pparg -J-040712-07 & UCACAAUGCCAUCAGGUUU \\
\hline & Pparg -J-040712-08 & CGACAUGAAUUCCUUAAUG \\
\hline \multirow[t]{4}{*}{ Cebpb (12608) } & Cebpb -J-043110-09 & GAGCGACGAGUACAAGAUG \\
\hline & Cebpb-J-043110-10 & CCUUUAGACCCAUGGAAGU \\
\hline & Cebpb-J-043110-11 & GCACCCUGCGGAACUUGUU \\
\hline & Cebpb-J-043110-12 & GAAAAGAGGCGUAUGUAUA \\
\hline $\begin{array}{l}\text { Non-targeting } \\
\text { siRNA \#2 }\end{array}$ & D-001810-02-05 & UGGUUUACAUGUUGUGUGA \\
\hline
\end{tabular}

cell lines (Fig. 1c, d). The expression of peroxisomeproliferator activated receptor $\gamma$ (PPAR $\gamma$ ), a critical regulator of adipogenesis, that is induced during differentiation, confirmed functional adipogenesis (Fig. 1c, d).

DDX5 is required for adipogenesis - Next, we investigated the consequences of supressing $D d x 5$ expression using siRNA. We obtained an $>80 \%$ reduction in transcript levels using siRNA targeted against $D d x 5$ in 3T3L1 cells but only a $\sim 55 \%$ reduction in C3H10T1/2 cells compared to cells treated with control siRNA (Fig. 1e, f). Cell viability was assessed to investigate if the $\operatorname{Ddx} 5$ knockdown impaired viability. We observed that Ddx5 knockdown did not significantly impair viability ( $>90 \%$ viability) when compared to untreated cells (Fig. 1g). Given the greater knockdown observed in 3T3-L1 cells, subsequent knockdown experiments were performed on 3T3-L1 cells to understand how the loss of $D d x 5$ affects adipogenesis. $D d x 5$ was suppressed throughout adipogenesis in a sustained and systematic manner by the addition of siRNA every 2 days starting from 2 days before the induction of adipogenesis to day 6 of differentiation (Fig.2a, d). We found that treatment with siRNA against $D d x 5$ resulted in impaired triglyceride accumulation compared to treatment with control siRNA, as observed by brightfield microscopy, Oil Red O staining and triglyceride quantification (Fig. 2b, c). Cells were also treated with siRNA against Pparg as a positive control, as it is well established that disruption of Pparg expression blocks triglyceride accumulation and adipogenesis [9]. Since $D d x 5$ suppression impairs triglyceride accumulation, 


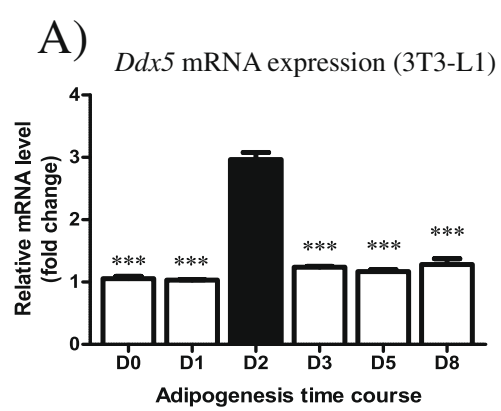

C) $\begin{array}{llllll}\text { D0 } & \text { D1 } & \text { D2 } & \text { D3 } & \text { D5 } & \text { D8 }\end{array}$
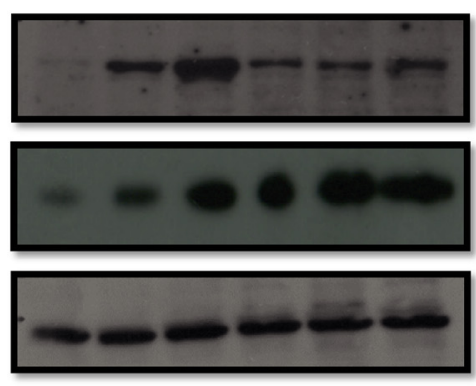

3T3-L1 cells

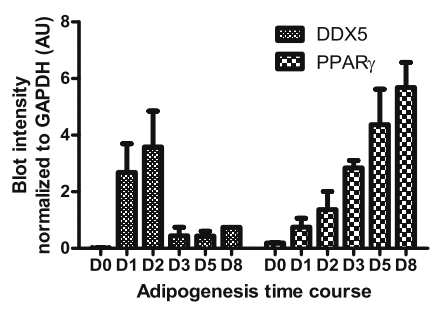

B)

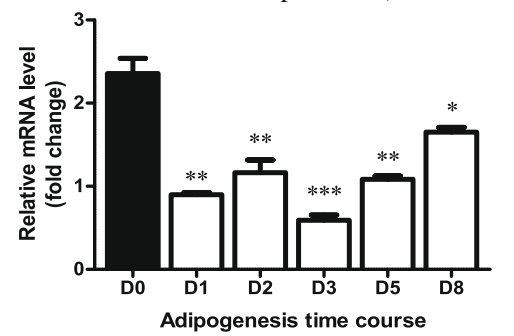

Protein D)

(kDa)

DDX5(68)

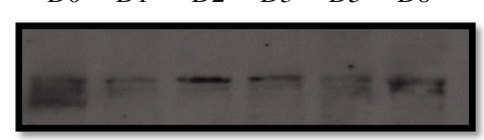

$\operatorname{PPAR} \gamma(54)$

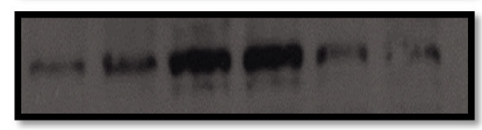

GAPDH(37)

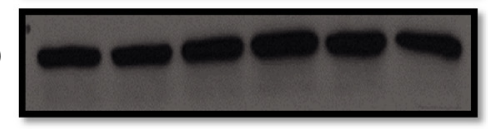

C3H10T1/2 cells

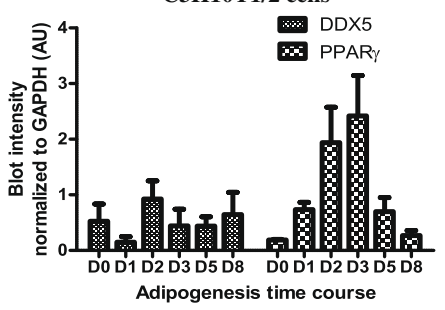

E)

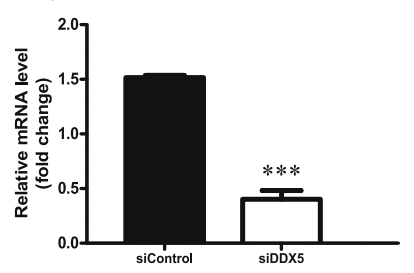

F) C3H10T1/2 siRNA knockdown

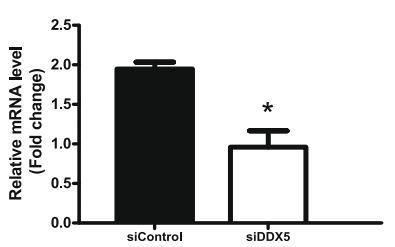

G) wST-1 Assay (Cell Viability)

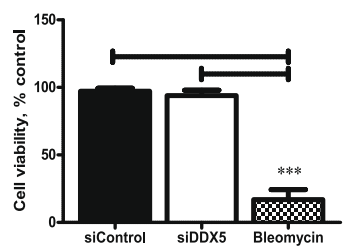

Fig. 1 mRNA and protein expression of DDX5 assayed in 3T3-L1 preadipocytes and C3H10T1/2 mesenchymal stem cells induced to undergo adipogenesis for 8 days in vitro. mRNA expression of Ddx5 in (a) 3T3-L1 and (b) C3H10T1/2 cells differentiated for 8 days in vitro. Black bars indicate the peak of mRNA expression. Protein expression by western blotting for DDX5, PPARY and GAPDH in (c) 3T3-L1 and (d) C3H10T1/2 cells differentiated for 8 days in vitro. Western blot bands were quantified using ImageJ. Transient knockdown using siRNA were performed on (e) 3T3-L1 and (f) C3H10T1/2 cells. g Cell viability assay using WST-1 was performed using cells treated with control siRNA (siControl), siRNA against Ddx5 (siDDX5) and bleomycin and compared to untreated cells. Data are represented as \% cell viability compared to untreated cells. ${ }^{*} p<0.05,{ }^{* *} p<0.01,{ }^{* *} p<0.001$. Experiments were done in triplicates and error bars indicate SEM $(n=3)$. mRNA expression data and western blot densitometry were normalized to CycloA and GAPDH expression respectively

we determined if this was due to impaired adipogenesis. We assessed mRNA expression of $D d x 5$, CCAAT enhancer binding protein $\beta(C e b p b)$, a transcription factor critical at the early stages of adipogenesis [9], Pparg, a critical regulator that maintains the mature adipocytic state [9], and Lmna whose expression is relatively stable during adipogenesis and in fully differentiated cells [10]. $D d x 5$ expression was significantly suppressed by siRNA treatment throughout adipogenesis as expected (Fig. 2d). Both Cebpb and Pparg expression were also significantly reduced 
A) siRNA addition (sustained)

dipogenic

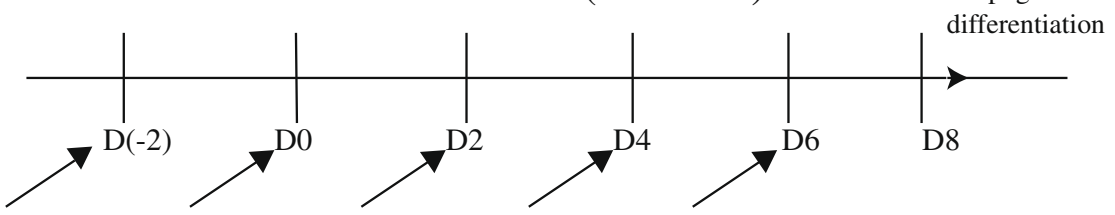

B)

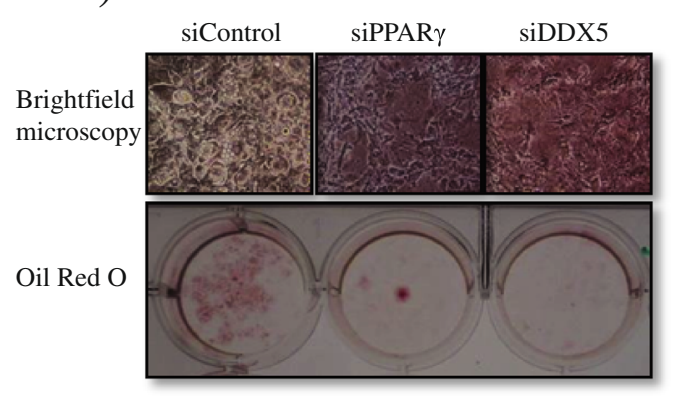

D)

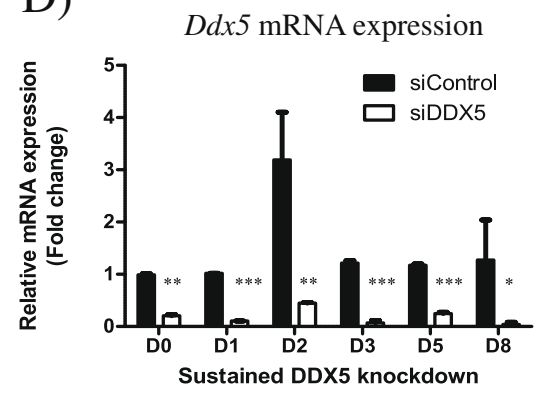

F)

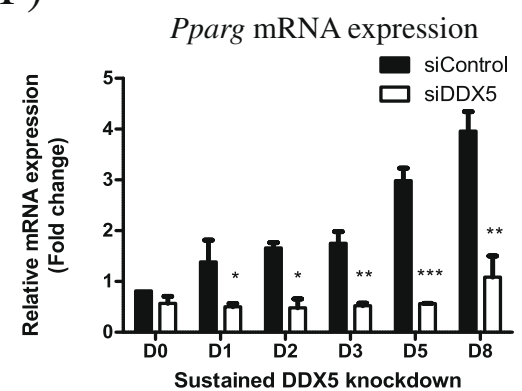

C)

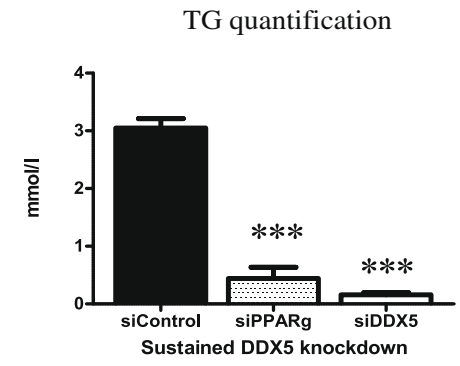

E)

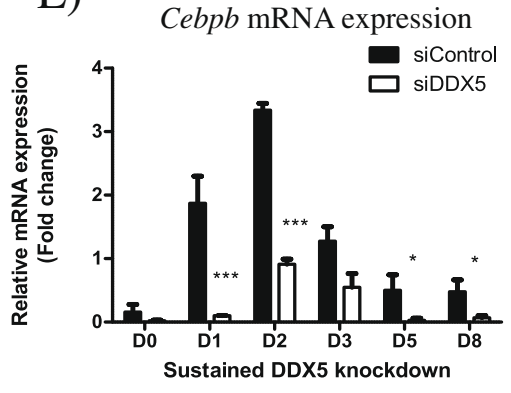

G)

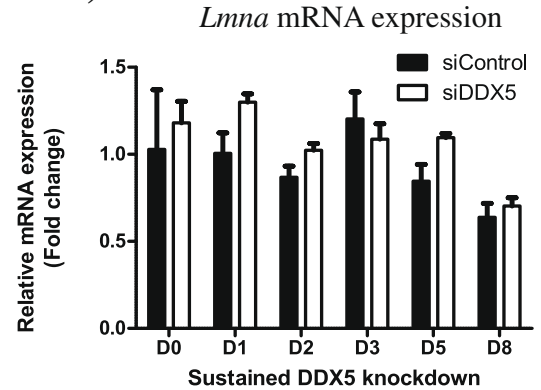

Fig. 2 Sustained knockdown of Ddx5 in 3T3-L1 cells differentiated for 8 days in vitro. (a) Cells were treated with siRNA continuously up to day 6 of differentiation and (b) viewed under a microscope and also stained for lipid accumulation using Oil Red O Stain. (c) Triglyceride accumulation was quantified and mRNA expression of (d) Ddx5, (e) Cebpb, (f) Pparg and (g) Lmna were investigated. All experiments were done in triplicates and statistical significance are denoted as follows: ${ }^{*} p<0.05,{ }^{* *} p<0.01,{ }^{* * *} p<0.001$. Error bars indicate SEM $(n=3)$. mRNA expression data were normalized to CycloA expression respectively

(Fig. 2e, f), suggesting that both these early and later regulators of adipogenesis are reduced by the knockdown of $D d x 5$. Lmna expression was unaffected by the $D d x 5$ inhibition (Fig. 2g) suggesting that the $D d x 5$ suppression particularly affects genes that regulate adipogenesis.
DDX5 is crucial at the early stages of adipogenesis Given the peak of expression at D2 of differentiation (Fig. 1a, c) and since $D d x 5$ is required for adipogenesis (Fig. 1e, f), we investigated the possibility of $D d x 5$ being crucial to the early stages of adipogenesis. We investigated 
this by transiently suppressing $D d x 5$ mRNA expression at the initial stages of adipogenesis: 2 days before adipogenic induction of adipogenesis (D-2) and at the same time as adipogenic induction (D0) (Fig. 3a, b). Differentiation was maintained for 8 days, however triglyceride accumulation was severely impaired, indicating defective adipogenesis (Fig. 3b, c). Cebpb is a well-established crucial factor in the early stages of adipogenesis [9], and was analyzed to further understand how the loss of $D d x 5$ affects early adipogenesis. While $D d x 5$ was only transiently suppressed at the early stages of differentiation (Fig. 3d), the expression of both Cebpb and more interestingly Pparg, a gene expressed much later in adipogenesis, were impaired compared to cells treated with control siRNA (Fig. 3e, f),

A)

\section{siRNA addition (early)}

dipogenic

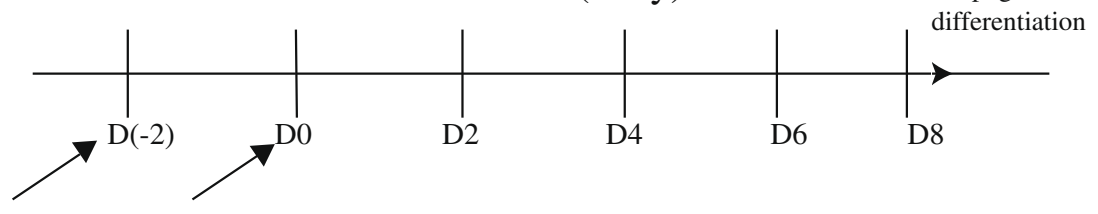

B)

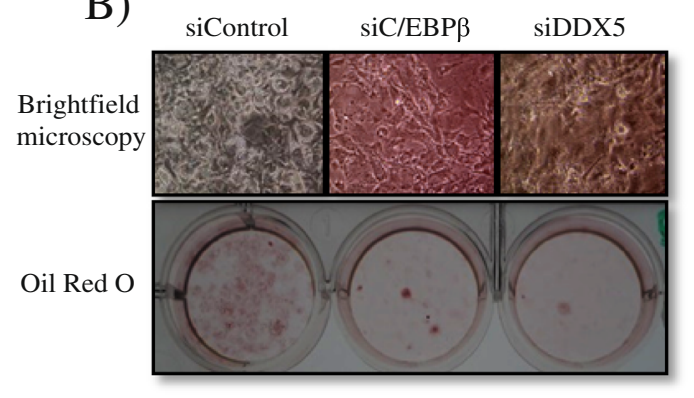

C)

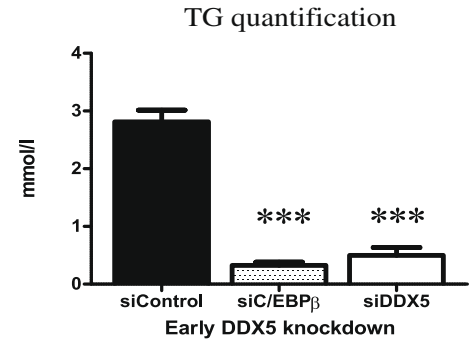

D)

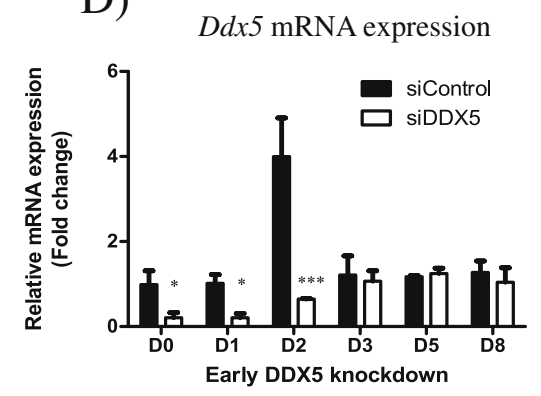

F)

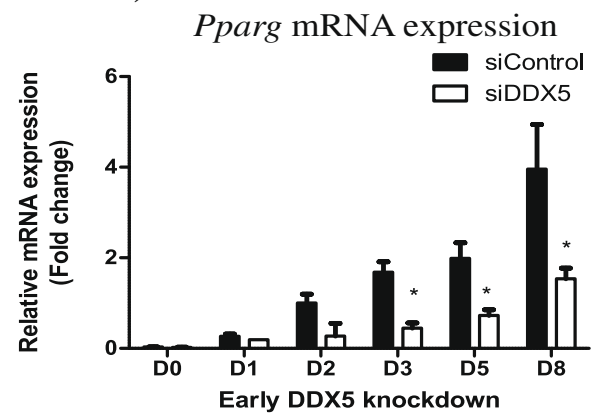

E)

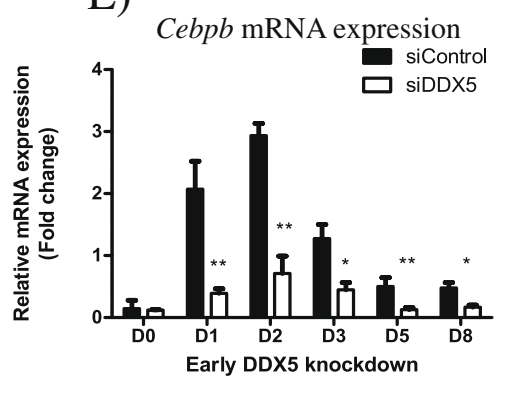

G)

Lmna mRNA expression

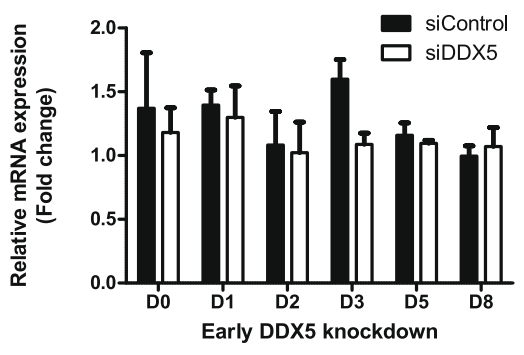

Fig. 3 Early knockdown of Ddx5 in 3T3-L1 cells differentiated for 8 days in vitro. (a) Cells were treated with siRNA only at D(-2) and D0 of differentiation and (b) viewed microscopically and also stained for lipid accumulation using Oil Red O stain. (c) Triglyceride accumulation was quantified and mRNA expression of (d) Ddx5, (e) Cebpb, (f) Pparg and (g) Lmna were investigated. All experiments were performed in triplicates and statistical significance are denoted as follows: ${ }^{*} p<0.05,{ }^{* *} p<0.01,{ }^{* * *} p<0.001$. Error bars indicate SEM $(n=3)$. mRNA expression data were normalized to CycloA expression respectively 
whereas the expression of Lmna was unaffected (Fig. 3g). Cebpb induces the expression of Pparg [9] and this may explain, at least in part, the suppression of Pparg expression.

DDX5 is not required in mature adipocytes - Since $D d x 5$ was required during the early stages of adipogenesis we investigated the requirement for DDX5 at the later stages of adipogenesis. Differentiating 3T3-LI adipocytes were treated with siRNA at days 4 and 6 of differentiation (Fig. 4a). Prior to this, the cells are differentiated but untreated and so indistinguishable experimentally. Thus data only from D4 onwards would be relevant and meaningful and only these are presented. In addition, analysis of $C e b p b$ is also omitted given that its expression is only relevant early in adipogenesis. Cells treated with siRNA
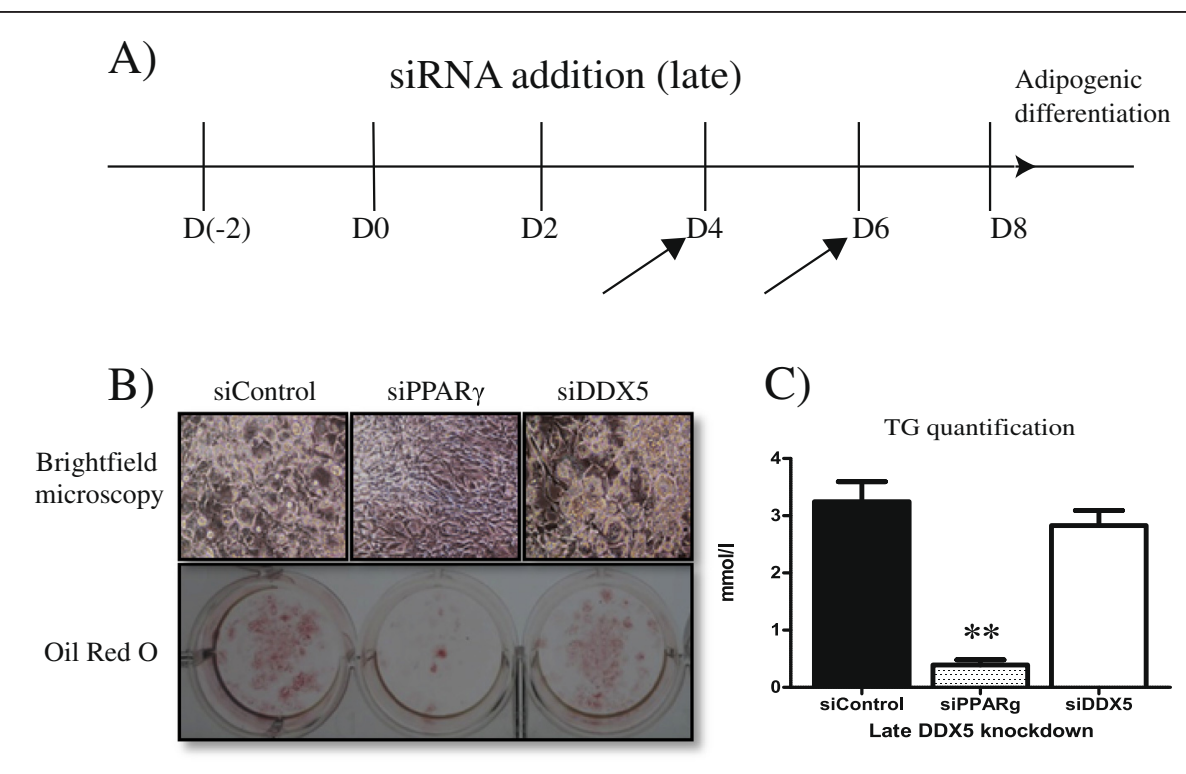

C)

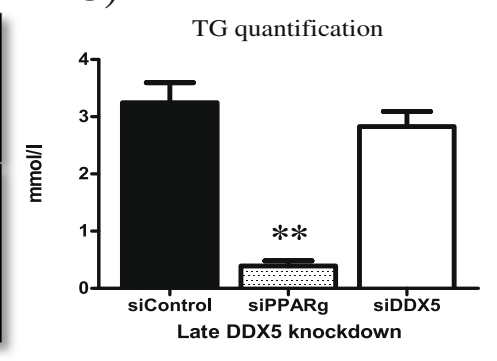

D)
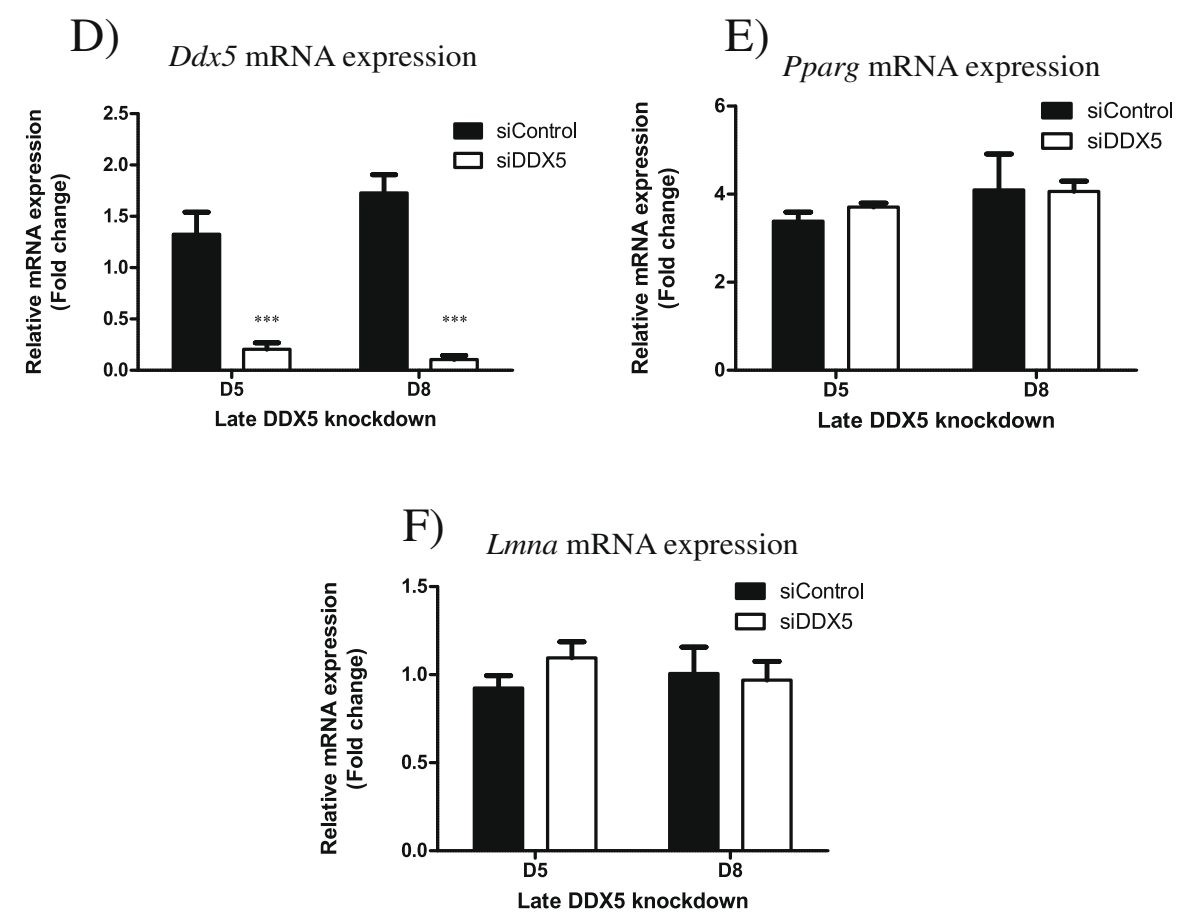

Fig. 4 Late knockdown of Ddx5 in 3T3-L1 cells differentiated for 8 days in vitro. (a) Cells were treated with siRNA only at D4 and D6 of differentiation and (b) viewed microscopically and also stained for lipid accumulation using Oil Red $O$ stain. (c) Triglyceride accumulation was quantified and mRNA expression of (d) Ddx5, (e) Pparg and (f) Lmna were described. All experiments were done in triplicates and statistical significance are denoted as follows: ${ }^{*} p<0.05,{ }^{* *} p<0.01$, ${ }^{* * *} p<0.001$. Error bars indicate SEM $(n=3)$. mRNA expression data were normalized to CycloA expression respectively 
targeting $D d x 5$ in this manner accumulated triglycerides as well as cells treated with control siRNA (Fig. 4b, c). However treatment with siRNA against Pparg impaired the maintenance of the mature adipocyte state, consistent with previous reports [11]. $D d x 5$ siRNA suppressed $D d x 5$ expression (Fig. 4d) but Pparg and Lmna levels were unaffected (Fig. 4e, f). Ddx5 therefore appears to be essential for the initial stages of adipogenesis, but not at the later stages of differentiation and maintenance of the mature adipocyte state.

\section{Discussion}

Here we demonstrate that DDX5/p68 RNA helicase peaks in expression on day 2 of adipogenesis in two different murine cell lines (Fig. 1a-d). These observations led us to speculate that this transient expression is required at the early stage of adipogenesis. The transcriptional pathways controlling adipocyte differentiation are relatively well characterized [12]. Adipogenesis is regulated by a cascade in the expression of a variety of transcription factors where an early wave of factors is induced immediately at the start of differentiation. These factors then activate a second set of transcription factors that are responsible in maintaining the mature and functional adipocyte. Utilising a transient knockdown strategy, we observed that DDX5 is essential during the initial stages of adipogenesis (Fig. 3) but not at later stages (Fig. 4). This suggests a role for DDX5, potentially as a transcriptional co-activator, at the early stages of adipocytic differentiation, placing it in the same category as other well established transcriptional factors such as $\mathrm{C} / \mathrm{EBP} \beta / \delta$ and the Kruppel-like factors (KLFs) [9]. In future studies it will be of interest to examine how the loss of Ddx 5 affects lipid droplet biology given the increasing evidence of genes expressing lipid droplet associated proteins being implicated in metabolic diseases such as lipodystrophy [13]. This unappreciated role of DDX5 in adipogenesis provides further insight into adipogenic molecular pathways, providing an additional potential target to address metabolic diseases including obesity and type 2 diabetes that are of increasing importance to global public health.

\section{Competing interests}

The authors declare no conflict of interest.

\section{Authors' contributions}

NR designed the experiments, carried out the gene expression and initial knockdown characterisation studies in Figs. 2, 3, and 4, and wrote the manuscript. NL was involved in the design of the experiments and performed the experiments and analyses described in Fig. 1. CLS conceived the study, participated in its design and coordination and wrote the final manuscript. All authors read and approved the final manuscript.

\section{Acknowledgements}

$N R$ and CLS are supported in part by the Singapore Biomedical Research Council and the Singapore Agency for Science, Technology and Research (A*STAR). NL is supported in part by Nanyang Technological University (NTU), Singapore and the Singapore Biomedical Research Council.

\section{Author details}

'Developmental and Regenerative Biology Laboratory, Institute of Medical Biology, \#06-06, 8A Biomedical Grove, Singapore 138648, Republic of Singapore. ${ }^{2}$ School of Biological Sciences, College of Science, Nanyang Technological University, Singapore 637551, Republic of Singapore. ${ }^{3}$ Present address: Davos Life Science Pte Ltd, 3 Biopolis Drive, \#04-19, Synapse, Singapore 138623, Republic of Singapore.

Received: 29 July 2015 Accepted: 1 December 2015

Published online: 04 December 2015

\section{References}

1. Lane DP, Hoeffler WK. SV40 large T shares an antigenic determinant with a cellular protein of molecular weight 68,000. Nature. 1980;288:167-70.

2. Guo J, Hong F, Loke J, Yea S, Lim CL, Lee U, et al. A DDX5 S480A polymorphism is associated with increased transcription of fibrogenic genes in hepatic stellate cells. J Biol Chem. 2010;285:5428-37.

3. Liu Z-R. p68 RNA helicase is an essential human splicing factor that acts at the U1 snRNA-5' splice site duplex. Mol Cell Biol. 2002;22:5443-50.

4. Fuller-Pace FV. The DEAD box proteins DDX5 (p68) and DDX17 (p72): multi-tasking transcriptional regulators. Biochim Biophys Acta - Gene Regul Mech. 1829;2013:756-63.

5. Kitamura A, Nishizuka M, Tominaga K, Tsuchiya T, Nishihara T, Imagawa M. Expression of p68 RNA helicase is closely related to the early stage of adipocyte differentiation of mouse 3T3-L1 cells. Biochem Biophys Res Commun. 2001;287:435-9.

6. Payne VA, Grimsey N, Tuthill A, Virtue S, Gray SL, Dalla Nora E, et al. The human lipodystrophy gene BSCL2/seipin may be essential for normal adipocyte differentiation. Diabetes. 2008;57:2055-60.

7. Ramanathan N, Ahmed M, Raffan E, Stewart CL, O'Rahilly S, Semple RK, et al. Identification and characterisation of a novel pathogenic mutation in the human lipodystrophy gene AGPAT2: C48R: a novel mutation in AGPAT2. JIMD Rep. 2013:9:73-80.

8. Sim MFM, Dennis RJ, Aubry EM, Ramanathan N, Sembongi H, Saudek V, et al. The human lipodystrophy protein seipin is an ER membrane adaptor for the adipogenic PA phosphatase lipin 1. Mol Metab. 2013;2:38-46.

9. Rosen ED, MacDougald OA. Adipocyte differentiation from the inside out. Nat Rev Mol Cell Biol. 2006;7:885-96.

10. Constantinescu D, Gray HL, Sammak PJ, Schatten GP, Csoka AB. Lamin A/C expression is a marker of mouse and human embryonic stem cell differentiation. Stem Cells. 2006;24:177-85.

11. He W, Barak Y, Hevener A, Olson P, Liao D, Le J, et al. Adipose-specific peroxisome proliferator-activated receptor gamma knockout causes insulin resistance in fat and liver but not in muscle. Proc Natl Acad Sci U S A. 2003; 100:15712-7.

12. Lowe CE, O'Rahilly S, Rochford JJ. Adipogenesis at a glance. J Cell Sci. 2011; 124:2681-6.

13. Robbins AL, Savage DB. The genetics of lipid storage and human lipodystrophies. Trends Mol Med. 2015;21:433-8.

Submit your next manuscript to BioMed Central and we will help you at every step:

- We accept pre-submission inquiries

- Our selector tool helps you to find the most relevant journal

- We provide round the clock customer support

- Convenient online submission

- Thorough peer review

- Inclusion in PubMed and all major indexing services

- Maximum visibility for your research

Submit your manuscript at www biomedcentral.com/submit

C Biomed Central 